\title{
Immunomodulation of human monocytes following exposure to
} Lutzomyia intermedia saliva

Maria José Menezes ${ }^{1}$, Dirceu J Costa ${ }^{1}$, Jorge Clarêncio ${ }^{1}$, José Carlos Miranda ${ }^{1}$, Aldina Barral1,2,3, Manoel Barral-Netto ${ }^{1,2,3}$, Cláudia Brodskyn ${ }^{1,2,3}$ and Camila I de Oliveira*1

\author{
Address: ${ }^{1}$ Centro de Pesquisas Gonçalo Moniz-FIOCRUZ, Salvador, Brazil, ${ }^{2}$ Universidade Federal da Bahia, Salvador, Brazil and ${ }^{3}$ Instituto de \\ Investigação em Imunologia, Salvador, Brazil \\ Email: Maria José Menezes - mjmenezes@usp.br; Dirceu J Costa - dirceu@bahia.fiocruz.br; Jorge Clarêncio - jorgec@bahia.fiocruz.br; \\ José Carlos Miranda - jmiranda@bahia.fiocruz.br; Aldina Barral - abarral@bahia.fiocruz.br; Manoel Barral-Netto - mbarral@bahia.fiocruz.br; \\ Cláudia Brodskyn - brodskyn@bahia.fiocruz.br; Camila I de Oliveira* - camila@bahia.fiocruz.br \\ * Corresponding author
}

Published: 10 April 2008

BMC Immunology 2008, 9:12 doi:10.1 I86//47|-2172-9-12
Received: 17 October 2007

Accepted: 10 April 2008

This article is available from: http://www.biomedcentral.com/I47I-2/72/9/12

(c) 2008 Menezes et al; licensee BioMed Central Ltd.

This is an Open Access article distributed under the terms of the Creative Commons Attribution License (http://creativecommons.org/licenses/by/2.0), which permits unrestricted use, distribution, and reproduction in any medium, provided the original work is properly cited.

\begin{abstract}
Background: Sand fly saliva contains potent and complex pharmacologic molecules that are able to modulate the host's hemostatic, inflammatory, and immune systems. In this study, we evaluated the effects of salivary gland sonicate (SGS) of Lutzomyia intermedia, the natural vector of Leishmania braziliensis, on monocytes obtained from the peripheral blood mononuclear cells (PBMC) of healthy volunteers. We investigated the effects of sand fly saliva on cytokine production and surface molecule expression of LPS-stimulated human monocytes uninfected or infected with L. braziliensis.

Results: Pre-treatment of non-infected human monocytes with L. intermedia SGS followed by LPSstimulation led to a significant decrease in IL- 10 production accompanied by a significant increase in CD86, CD80, and HLA-DR expression. Pre-treatment with SGS followed by LPS stimulation and $L$. braziliensis infection led to a significant increase in TNF- $\alpha$, IL-6, and IL- 8 production without significant alterations in co-stimulatory molecule expression. However, pre-treatment with $L$. intermedia SGS did not result in significant changes in the infection rate of human monocytes.
\end{abstract}

Conclusion: Our data indicate that $L$. intermedia saliva is able to modulate monocyte response, and, although this modulation is dissociated from enhanced infection with $L$. braziliensis, it may be associated with successful parasitism.

\section{Background}

Leishmaniasis is a protozoan parasitic infection transmitted by sand flies. Different species of Leishmania are associated with distinct clinical forms of disease. Cutaneous leishmaniasis (CL) caused by Leishmania major is usually benign; infection of human hosts leads to the development of a localized cutaneous lesion that eventually heals, leading to the generation of life long-immunity. In contrast, CL caused by L. braziliensis is distinguished from other leishmaniasis by its chronicity, latency, and tendency to metastasize in the human host [1]. In this disease, a single ulcer with elevated borders and a necrotic centre is frequently observed, and a chronic inflammatory response develops despite the paucity of parasites. In 
$1-5 \%$ of patients, muco-cutaneous leishmaniasis may occur due to the intrinsic ability of L. braziliensis to persist within lesion scars after spontaneous or chemotherapymediated healing and its ability to metastasize to the nasal mucosa [2-4].

Leishmania parasites are transmitted to the vertebrate host when the sand fly probes for a blood meal. During blood feeding, sand fly saliva, which contains a great variety of hemostatic, inflammatory, and immunomodulatory molecules (rev. in [5]), is injected into the host's skin. Sand fly saliva is also known to facilitate parasite survival [6-9]. The enhancing effects of sand fly saliva on disease development are associated with its ability to inhibit several functions of antigen presenting cells (APCs) such as antigen presentation and nitric oxide (NO) production [1012]. We have recently shown, using an experimental model of infection, that pre-exposure to Lutzomyia intermedia saliva is able to enhance infection with Leishmania braziliensis [13]. Importantly, individuals with active cutaneous leishmaniasis also showed higher humoral immune responses to $L$. intermedia saliva compared to control subjects, suggesting an association between positive anti-saliva immune response and the development of disease. Therefore, we were interested in determining the effects of $L$. intermedia saliva in a more restricted experimental system, employing LPS-stimulated human monocytes alone or infected with Leishmania braziliensis. This approach has already been undertaken with $P$. papatasi [14] and with L. longipalpis [15], with which it was shown that saliva impairs cytokine production and co-stimulatory molecule expression on human monocytes and dendritic cells. Monocytes and dendritic cells are present in the hemorrhagic pool created by sand fly feeding and are, therefore, exposed to sand fly saliva. Because of this, we have examined the effects of saliva from $L$. intermedia, the main vector of L. braziliensis in Brazil, on human monocytes stimulated with LPS and uninfected or infected with L. brazilienis. Understanding how salivary products interfere with LPS-induced co-stimulatory molecule expression, cytokine production, and parasite infection may help clarify the complex vector-parasite-human host interplay.

\section{Results and Discussion}

Within their saliva, sand flies have evolved an array of potent pharmacological components which induce a favorable microenvironment for adequate blood feeding and which may also be important for parasite establishment (rev. in [16]). L. intermedia is the sand fly species responsible for the transmission of Leishmania braziliensis, the causative agent of cutaneous and mucosal leishmaniasis; thus, we have investigated the effects of $L$. intermedia saliva on the host's immune response in terms of surface molecule expression and cytokine production. Initially, we found that human monocytes stimulated with L. intermedia SGS alone did not secrete detectable levels of IL12 p40 or of TNF- $\alpha$ (data not shown). For that reason, we opted to set up an experimental system in which human monocytes were pre-treated with $L$. intermedia SGS and were then stimulated with LPS. In cultures that were pretreated with SGS, we observed a significant decrease in IL10 production without significant changes in terms of IL6 , TNF- $\alpha$ or IL-12p40 production (Fig. 1). Modulation of cytokine profiles following exposure to sand fly SGS has already been described; $P$. papatasi saliva was shown to decrease the production of type 1 cytokines and to enhance the production of IL- 6 by monocytes [14]. Sand fly saliva from three different species was able to suppress the mitogen-induced proliferative response of murine spleen cells [17]. Last, Costa et al. [15] showed, using the same experimental system employed herein, that L. longipalpis SGS inhibits TNF- $\alpha$ and IL-10 production while augmenting IL-6, IL-8, and IL-12p40 production. In that work, it was proposed that components in that sand-fly saliva may promote the development of a cell-mediated immune response through the up-regulation of IL-12 and inhibition of IL-10 production. To that end, development of a delayed-type hypersensitivity (DTH) response was associated with protection against leishmaniasis induced by immunization with SP15, a protein present in $P$. papatasi saliva [18].

Co-stimulatory molecules play an essential role in the activation and maintenance of T-cell responses. We thus analyzed whether pre-exposure to $L$. intermedia saliva had any effect on CD80, CD86, and HLA-DR expression. Monocytes were pre-treated with SGS and stimulated with LPS, and surface molecule expression was analyzed by flow cytometry. Surprisingly, pre-treatment with SGS was able to significantly increase the mean fluorescence intensity (MFI) of CD80, CD86, and HLA-DR on human monocytes (Fig. 2). Such up-regulation of co-stimulatory molecule expression was not observed with $L$. longipalpis SGS [15], indicating once again that salivary components of these two sand fly species induce different immunomodulatory effects. We can speculate that the increase in co-stimulatory molecule expression observed with $L$. intermedia saliva may be related to the decreased levels of IL-10 (Fig. 1) since it was shown that interleukin-10 differentially regulates B7-1 (CD80) and B7-2 (CD86) expression on human peripheral blood dendritic cells [19]. Class II MHC molecules are required for the activation of CD4 T cells and protection against L. major [20]. CD80, CD86, and CTLA-4 also influence the immune response to Leishmania $[21,22]$. The increased expression of co-stimulatory molecules also enhances antigen presentation that, ultimately, may lead to increased T cell activation and IFN- $\gamma$ production. Indeed, cutaneous leishmaniasis is associated with an intense inflammatory 

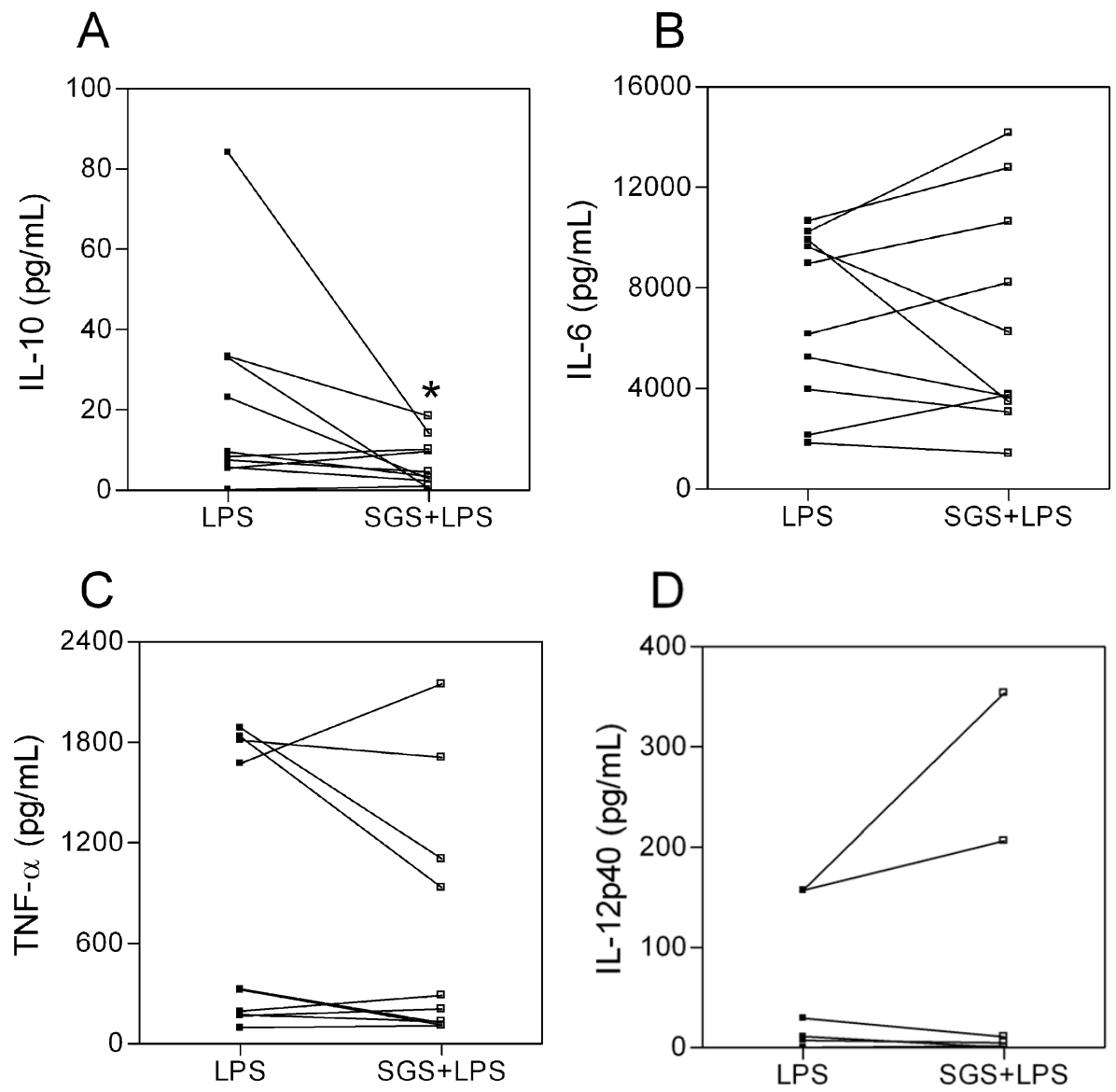

\section{Figure I}

Effect of $L$. intermedia SGS on LPS-stimulated human monocytes. Monocytes were pre-treated with SGS overnight and stimulated with LPS for $24 \mathrm{~h}$ (TNF- $\alpha$ and IL-6) or $48 \mathrm{~h}$ (IL- 10 and IL-I 2p40). The presence of IL-I0 (A), IL-6 (B), TNF- $\alpha$ (C) and IL-I2p40 (D) was analyzed by ELISA. The data for individual donors are presented. $\left({ }^{*} \mathrm{p}<0.05\right) .(\mathrm{n}=10)$

reaction $[23,24]$. Given these results, we then investigated whether the immune modulation exerted by $L$. intermedia saliva would affect parasite load upon monocyte infection with L. braziliensis. To address this hypothesis, human monocytes were pre-treated with $L$. intermedia saliva, stimulated with LPS, and then infected with $L$. braziliensis. Notably, we observed a significant increase in TNF- $\alpha$, IL6 , and IL-8 production upon parasite infection in cultures pre-treated with SGS, whereas IL-10 levels did not change (Fig. 3). We did not detect significant differences in CD80, HLA-DR (Fig. 4) or in CD86 expression (data not shown). However, up-regulation of the production of TNF- $\alpha$, IL-6, and IL-8 following pre-treatment with $L$. intermedia SGS and $L$. braziliensis infection did not change the parasite load as measured by the number of infected monocytes (Fig. 5A) or by the number of amastigotes per infected monocyte (Fig. 5B).
Norsworthy et al. [9] also failed to detect significant changes in the infection rate of $\mathrm{L}$. amazonensis in murine macrophages that were pre-treated with $\mathrm{L}$. longipalpis saliva, despite the significantly higher IL-10 mRNA levels observed. However, addition of Maxadilan, the potent vasodilatory peptide present in $\mathrm{L}$. longipalpis saliva, alone was able to up-regulate IL-10, IL- 6 and TGF- $\beta$ and to increase the L. major parasite load [25]. We are currently investigating $\mathrm{L}$. intermedia and $\mathrm{L}$. longipalpis saliva in terms of differences or similarities in immunomodulatory compounds and preliminary results indicate that Maxadilan is not abundant in L. intermedia saliva (de Oliveira et al., manuscript in preparation). SDS-PAGE profiles of salivary proteins from both sand fly species showed bands migrating at similar molecular weights, although L. intermedia immune sera does react with any protein present in L. longipalpis SGS with the exception of the $\sim 45 \mathrm{kDa}$ pro- 

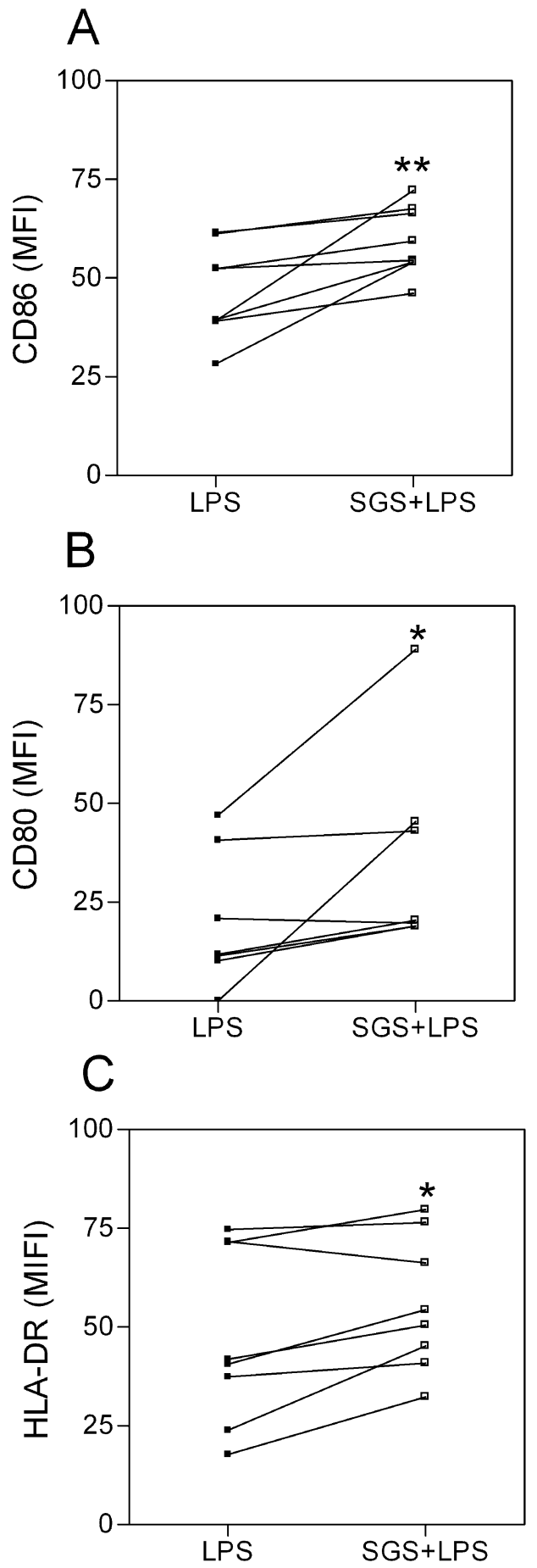

Figure 2

Effect of $L$. intermedia SGS on co-stimulatory molecule expression on LPS-stimulated human monocytes. Monocytes were pre-treated with SGS and stimulated with LPS for $48 \mathrm{~h}$. Cells were collected and surface molecule expression was analyzed by flow cytometry. Mean fluorescence intensity of expression is shown for each individual tested $\left(*_{p}<0.05\right) .(n=8)$. tein [13], indicating differences in the immunogenicity of salivary molecules.

It is well known that Th1 mediated immunity is important for the control of Leishmania infection and that oxidants produced by IFN- $\gamma$ activated macrophages are the main final effector molecules killing Leishmania (rev. in [26]. Although, in this report, we observed an increased production of TNF- $\alpha$ upon pre-treatment of monocytes with L. intermedia SGS, this was not associated with decreased L. braziliensis infection. This effect may be explained by the parallel up-regulation of both IL- 6 and IL-8 production. IL-6 is produced by cells of both the innate and adaptive immune responses, and it was recently shown that IL- 6 is able to drive the differentiation of $\mathrm{CD} 4{ }^{+} \mathrm{Th} 2$ cells by the early induction of IL- 4 in CD4+ $\mathrm{T}$ cells [27]. IL-8 guides polymorphonuclear cell recruitment to the site of infection, and neutrophils have been shown to act as "Trojan horses" [28] for Leishmania establishment. Indeed, development of clinically evident lesions occurs in parallel with the influx of inflammatory cells, including neutrophils, eosinophils and macrophages [29]. Using the air pouch model of inflammation, we observed a significant increase in the recruitment of polymorphonuclear cells upon injection of $L$. intermedia SGS (de Moura et al., manuscript in preparation). In mice exposed to $L$. intermedia SGS, a further inoculation of $L$. intermedia SGS induced an important inflammatory response comprised of numerous polymorphonuclear and few mononuclear cells [13]. We can thus hypothesize that the increase in IL-8 production induced by $L$. intermedia saliva may lead to neutrophil recruitment and, consequently, the successful establishment of infection. Moreover, it will also be interesting to determine whether $L$. intermedia saliva also exerts effects on the effector functions of T-lymphocytes, employing, for example in vitro priming systems $[30,14]$.

\section{Conclusion}

We can conclude that the effects exerted by $L$. intermedia saliva on human monocytes are different from those observed with L. longipalpis saliva [15], Maxadilan alone $[31,25]$ and $P$. papatasi saliva [14] and such differences may play an important role regarding the outcome of leishmaniasis. Moreover, since it has been proposed that vaccinating against sand fly salivary components can protect the host from infection (rev. in [32]), our observations also suggest a note of caution regarding the development of vaccines based on sand fly saliva.

\section{Methods}

Sand Flies and Preparation of SGS

Lutzomyia intermedia, Corte de Pedra strain, and Lutzomyia longipalpis, Cavunge strain, were reared at Centro de Pesquisas Gonçalo Moniz-FIOCRUZ, as described elsewhere [33]. Adult sand flies were used for dissection of 

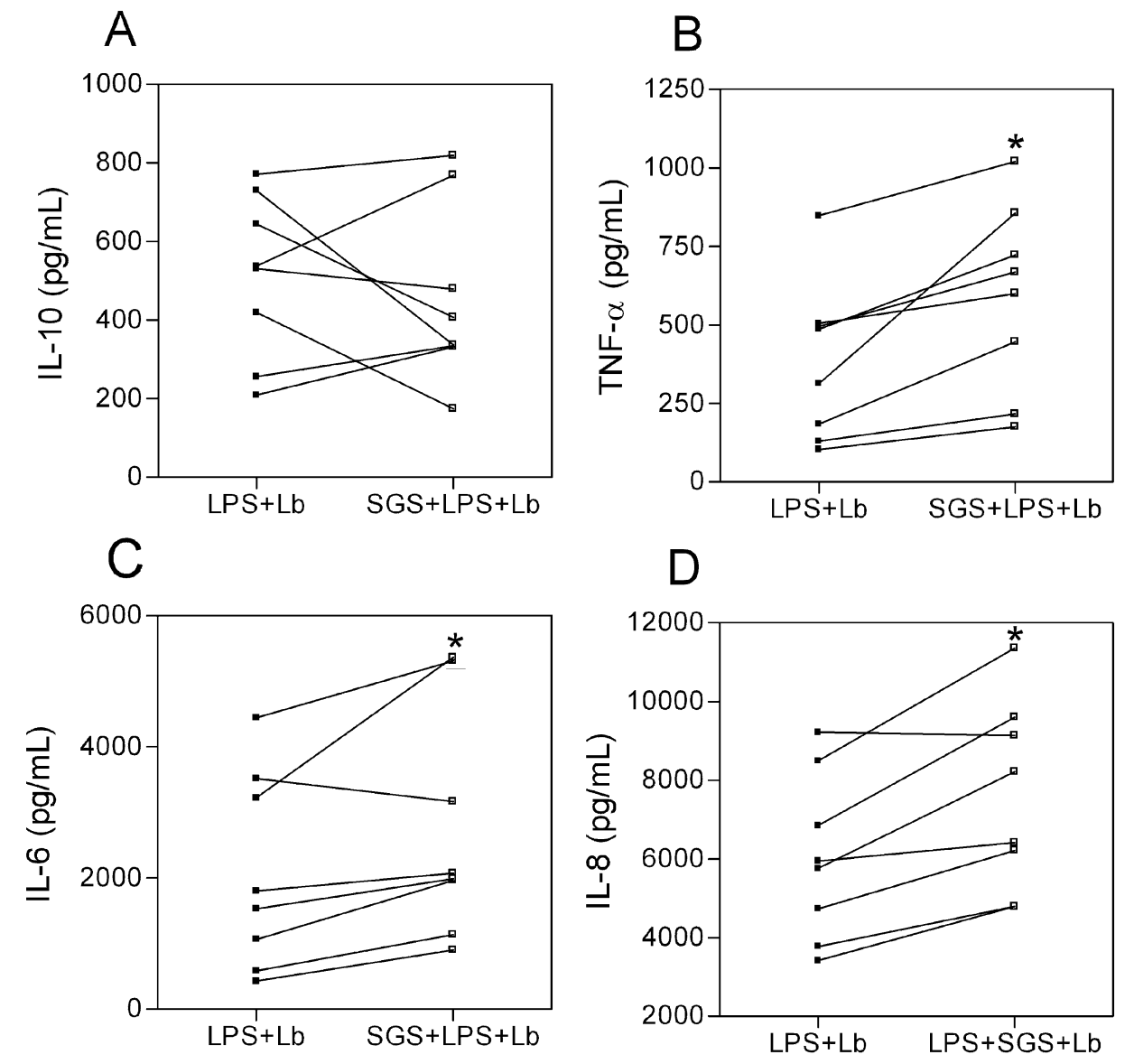

\section{Figure 3}

Effect of $L$. intermedia SGS on cytokine production of LPS-stimulated human monocytes infected with $L$. braziliensis. Monocytes were pre-treated with SGS overnight, stimulated with LPS for $4 \mathrm{~h}$ and infected with $L$. braziliensis for 4 hours. The presence of IL-I0 (A), TNF- $\alpha$ (B), IL-6 (C) and IL-8 (D) was analyzed by ELISA. The data for individual donors are presented. $\left({ }^{*} p<0.05\right) .(n=8)$.

salivary glands 3-5 days after emergence. Salivary glands were stored in groups of 20 pairs in $20 \mu \mathrm{l} \mathrm{NaCl}(150 \mathrm{mM})$ Hepes buffer $(10 \mathrm{mM}, \mathrm{pH} 7.4)$, at $-70^{\circ} \mathrm{C}$. Immediately before use, salivary glands were disrupted by ultrasonication in $1.5 \mathrm{ml}$ conical tubes. Tubes were centrifuged at $10,000 \times \mathrm{g}$ for $2 \mathrm{~min}$ and the resultant supernatant (Salivary Gland Sonicate - SGS) was used for the studies. The level of LPS contamination of SGS preparations was determined using a commercially available LAL Chromogenic Kit (QCL-1000, Lonza Bioscience); LPS concentration was $<0.1 \mathrm{ng} / \mathrm{ml}$.

\section{Monocyte Culture}

In this work, leukocyte concentrates from healthy adult blood donors were provided by the Blood Bank (HEMOBA) from Salvador, Bahia. Informed verbal con- sent was obtained from blood donors (six male, four female, age range 30 to 39 years old)

at the time of donation, and all procedures were approved by the local Ethics Committee (CEP/CPqGM-FIOCRUZ) and were conducted following recommendations outlined in the Helsinki Declaration. PBMC were isolated by Ficoll-Hypaque (Sigma) density gradient separation. Cells were then washed, and monocytes were obtained by positive selection with CD14 magnetic beads (Miltenyi Biotech), following the manufacturer's instructions. Briefly, a total of $10^{8}$ cells were resuspended in MACS buffer (PBS/ $0.5 \% \mathrm{BSA} / 2 \mathrm{mM}$ EDTA) containing CD14 microbeads. After incubation for $30 \mathrm{~min}$ at $4{ }^{\circ} \mathrm{C}$, cells were washed and resuspended in MACS buffer and loaded onto MiniMACS MS+ Separation Columns (Miltenyi Biotech). Non-adherent cells were recovered and the total number of CD14- 

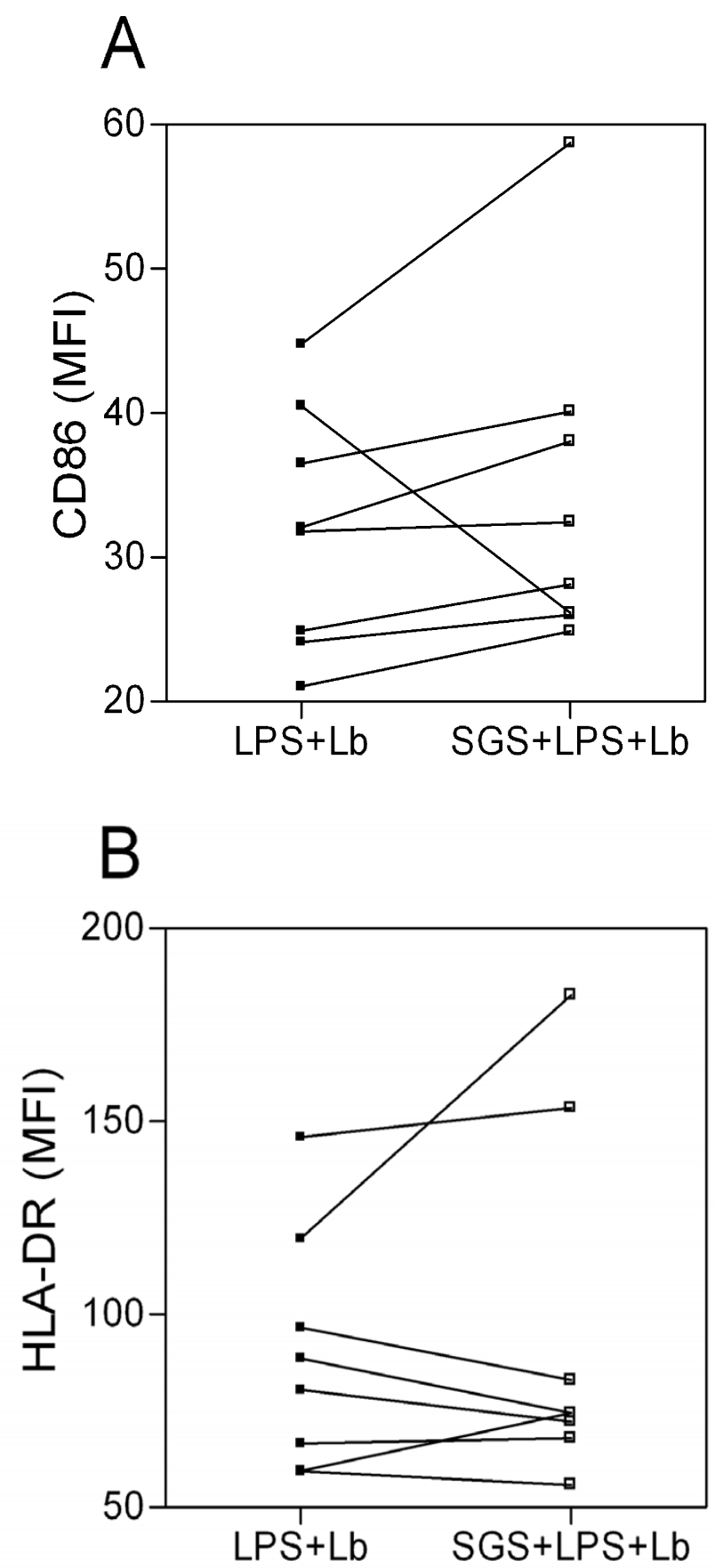

Figure 4

Effect of $L$. intermedia SGS on co-stimulatory molecule expression of LPS-stimulated human monocytes infected with $L$. braziliensis. Monocytes were pre-treated with SGS overnight, stimulated with LPS for $4 \mathrm{~h}$ and infected with L. braziliensis for 4 hours. Cells were collected $48 \mathrm{~h}$ later and surface molecule expression was analyzed by flow cytometry. Mean fluorescence intensity of expression is shown for each individual tested $\left({ }^{*} p<0.05\right)$. $(n=8)$. positive monocytes was determined by FACS. The average yield was usually between 92 and $98 \%$. Purified human monocytes were resuspended in complete RPMI (RPMI supplemented with HEPES Buffer 11203, L-glutamine (2 $\mathrm{mM})$, penicillin (100 U/mL), streptomycin (1\%) and fetal calf serum (10\%), all from Life Technologies) and were pre-treated with $L$. intermedia SGS (equivalent to 2 pairs of salivary gland/ml or $1 \mathrm{ug} / \mathrm{ml}$ of protein) for $12 \mathrm{~h}$, followed by stimulation with $20 \mathrm{pg} / \mathrm{ml}$ LPS (E. coli O111:B14 from Sigma). Cultured cells and culture supernatants were collected $24 \mathrm{~h}$ and $48 \mathrm{~h}$ later. Co-stimulatory molecule expression was analyzed by flow cytometry and cytokine profiles were determined by ELISA.

\section{Flow cytometry}

Reagents for staining cell surface markers and intracellular cytokines were purchased from BD Biosciences, San Diego, CA. Cells were blocked with anti-Fc receptor antibody (2.4G2) and were stained with anti-human CD80 (L307.4), CD86 (2331) and HLA-DR, DP and DQ (G466) conjugated to PE. Isotype controls were used as appropriate. For each sample, 20,000 events were analyzed using CELLQuest ${ }^{\mathrm{TM}}$ software and a FACSort ${ }^{\circledR}$ flow cytometer (Becton Dickinson Immunocytometry).

\section{Cytokine assays}

Concentrations of TNF- $\alpha$, IL-6, IL-8, IL-10 and IL-12p40 in culture supernatants were determined by ELISA using commercial kits (BD Pharmingen), following the manufacturer's instructions.

\section{Parasite culture}

L. braziliensis strain MHOM/BR/01/BA788 was isolated from a patient with cutaneous leishmaniasis from the state of Bahia (northeastern Brazil) after brief (2-4) passages in culture medium. This isolate was identified as $L$. braziliensis by using PCR [34] and monoclonal antibodies [35]. Promastigotes were grown in Schneider medium (Sigma) supplemented with $100 \mathrm{U} / \mathrm{ml}$ of penicillin, 100 $\mathrm{ug} / \mathrm{ml}$ of streptomycin and $10 \%$ heat-inactivated fetal calf serum (all from Life Technologies). Monocytes were pretreated with SGS as above for twelve hours, stimulated with LPS for four hours and were infected with $L$. braziliensis parasites ( 5 parasites to 1 monocyte) for four hours. Infected monocytes were washed for removal of parasites and cultivated for 24 and $48 \mathrm{~h}$. Co-stimulatory molecule expression was analyzed by flow cytometry and cytokine profiles were determined by ELISA.

\section{Statistical analysis}

Statistical analyses comparing cytokine responses and surface molecule expression were performed using Prism (GraphPad Software). Data were compared using the Wilcoxon signed-rank test. In all cases, results were considered significantly different when the $P$-value was $<0.05$. 

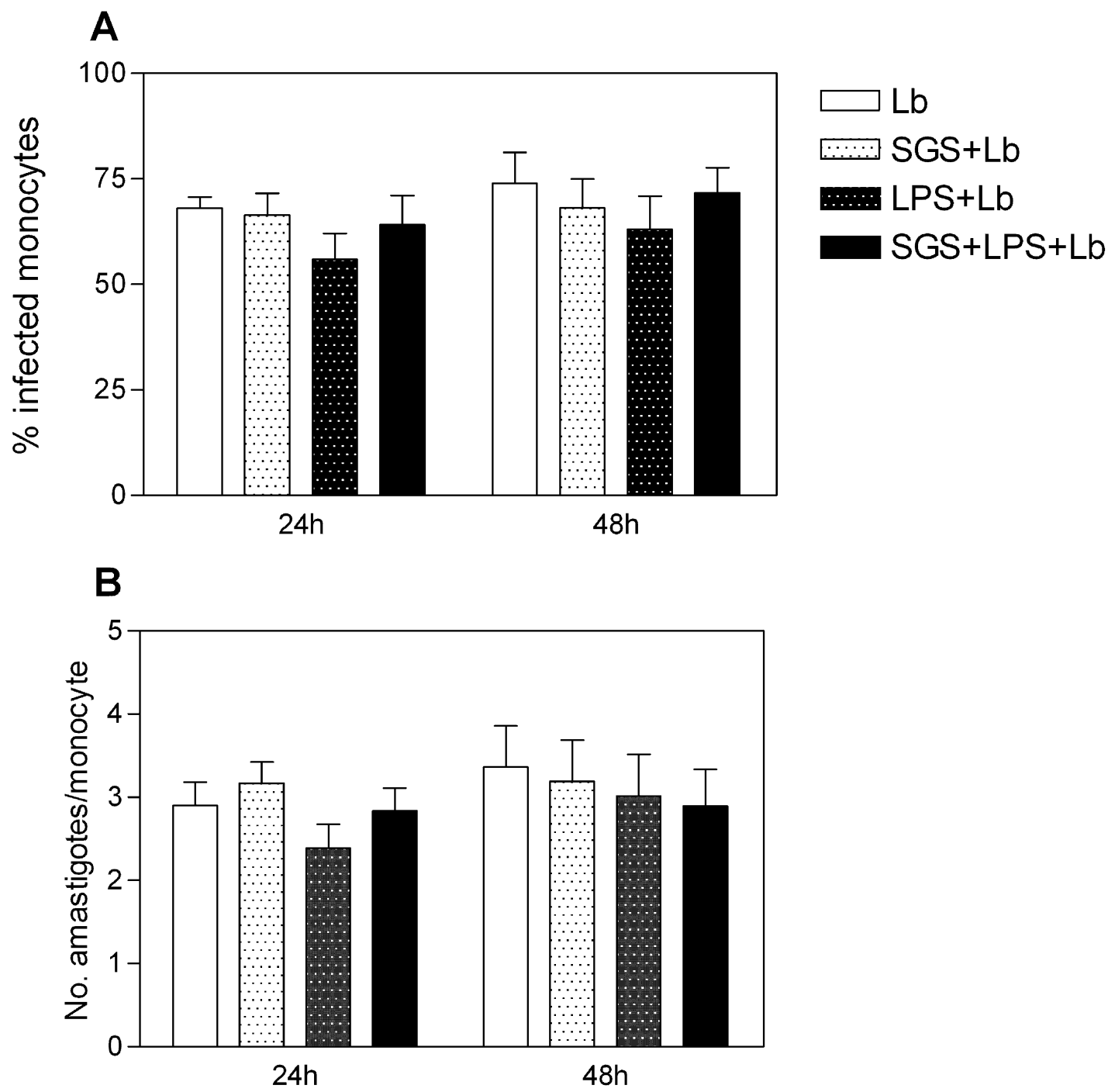

Figure 5

Effect of $L$. intermedia SGS on parasite load of LPS-stimulated human monocytes infected with $L$. braziliensis. Monocytes were pre-treated with SGS overnight, stimulated with LPS for $4 \mathrm{~h}$ and infected with L. braziliensis for 4 hours. Glass coverslips were stained with H\&E and counted for the number of infected cells $(A)$ and amastigotes per 100 cells (B) by light microscopy. The data are reported as the mean \pm the standard error of the mean. $(n=8)$.

\section{Authors' contributions}

MJM and JC performed experiments and analyzed data. JC carried out flow cytometry analysis. JCM contributed materials to the study. $\mathrm{AB}$ and MBN participated in study design and helped to draft the manuscript. $\mathrm{CB}$ participated in study design and data analysis. CIO conceived of the study, and participated in its design and coordination and drafted the manuscript. All authors read and approved the final manuscript.

\section{Acknowledgements}

This work was supported by grants from CNPq, FAPESB and PAPES/ FIOCRUZ. M.J.M. was supported by a CAPES fellowship; D.J.C was sup- ported by a CNPq fellowship. A.B.; M.B.N; C.B. and C.I.O. are senior investigators from CNPq and Instituto de Investigação em Imunologia (iii). We gratefully acknowledge the technical assistance of Edvaldo Passos.

\section{References}

I. Bittencourt A, Barral-Netto M: Leishmaniasis. In Tropical Pathology Volume 8. 2nd edition. Edited by: Doerr W SG. Springer, Berlin; 1995:597-65I.

2. Aebischer T, Moody SF, Handman E: Persistence of virulent Leishmania major in murine cutaneous leishmaniasis: a possible hazard for the host. Infect Immun 1993, 6I (I):220-226.

3. Schubach A, Marzochi MC, Cuzzi-Maya T, Oliveira AV, Araujo ML, Oliveira AL, Pacheco RS, Momen H, Conceicao-Silva F, Coutinho SG, Marzochi KB: Cutaneous scars in American tegumentary leishmaniasis patients: a site of Leishmania (Viannia) braziliensis persistence and viability eleven years after antimo- 
nial therapy and clinical cure. Am J Trop Med Hyg 1998, 58(6):824-827.

4. Mendonca MG, de Brito ME, Rodrigues EH, Bandeira V, Jardim ML, Abath FG: Persistence of leishmania parasites in scars after clinical cure of American cutaneous leishmaniasis: is there a sterile cure? J Infect Dis 2004, I 89(6): I018-1023.

5. Ribeiro JM: Blood-feeding arthropods: live syringes or invertebrate pharmacologists? Infect Agents Dis 1995, 4(3): 143-152.

6. Titus RG, Ribeiro JM: Salivary gland lysates from the sand fly Lutzomyia longipalpis enhance Leishmania infectivity. Science 1988, 239(4845): I306-1308.

7. Samuelson J, Lerner E, Tesh R, Titus R: A mouse model of Leishmania braziliensis braziliensis infection produced by coinjection with sand fly saliva. J Exp Med I99I, I 73(I):49-54.

8. Belkaid Y, Kamhawi S, Modi G, Valenzuela J, Noben-Trauth N, Rowton E, Ribeiro J, Sacks DL: Development of a natural model of cutaneous leishmaniasis: powerful effects of vector saliva and saliva preexposure on the long-term outcome of Leishmania major infection in the mouse ear dermis. J Exp Med 1998, 188(10):1941-1953.

9. Norsworthy NB, Sun J, Elnaiem D, Lanzaro G, Soong L: Sand fly saliva enhances Leishmania amazonensis infection by modulating interleukin-10 production. Infect Immun 2004, 72(3): 1240-1247.

10. Hall LR, Titus RG: Sand fly vector saliva selectively modulates macrophage functions that inhibit killing of Leishmania major and nitric oxide production. I Immunol 1995, I55(7):350I-3506.

II. Theodos CM, Titus RG: Salivary gland material from the sand fly Lutzomyia longipalpis has an inhibitory effect on macrophage function in vitro. Parasite Immunol 1993, I 5(8):48|-487.

12. Waitumbi J, Warburg A: Phlebotomus papatasi saliva inhibits protein phosphatase activity and nitric oxide production by murine macrophages. Infect Immun 1998, 66(4): $1534-1537$.

13. de Moura TR, Oliveira F, Novais FO, Miranda JC, Clarencio J, Follador I, Carvalho EM, Valenzuela JG, Barral-Netto M, Barral A, Brodskyn C, de Oliveira Cl: Enhanced Leishmania braziliensis Infection Following Pre-Exposure to Sandfly Saliva. PLoS neglected tropical diseases 2007, I (2):e84.

14. Rogers KA, Titus RG: Immunomodulatory effects of Maxadilan and Phlebotomus papatasi sand fly salivary gland lysates on human primary in vitro immune responses. Parasite Immunol 2003, 25(3): $127-134$

15. Costa DJ, Favali C, Clarencio J, Afonso L, Conceicao V, Miranda JC, Titus RG, Valenzuela J, Barral-Netto M, Barral A, Brodskyn Cl: Lutzomyia longipalpis salivary gland homogenate impairs cytokine production and costimulatory molecule expression on human monocytes and dendritic cells. Infect Immun 2004, 72(3):1298-1305.

16. Andrade BB, de Oliveira $\mathrm{Cl}$, Brodskyn Cl, Barral A, Barral-Netto M: Role of sand fly saliva in human and experimental leishmaniasis: current insights. Scandinavian journal of immunology 2007, 66(2-3): $122-127$.

17. Rohousova I, Volf P, Lipoldova M: Modulation of murine cellular immune response and cytokine production by salivary gland lysate of three sand fly species. Parasite Immunol 2005, 27(12):469-473.

18. Valenzuela JG, Belkaid Y, Garfield MK, Mendez S, Kamhawi S, Rowton ED, Sacks DL, Ribeiro JM: Toward a defined anti-Leishmania vaccine targeting vector antigens: characterization of a protective salivary protein. J Exp Med 200I, I 94(3):33I-342.

19. Buelens C, Willems F, Delvaux A, Pierard G, Delville JP, Velu T, Goldman M: Interleukin-I0 differentially regulates B7-I (CD80) and B7-2 (CD86) expression on human peripheral blood dendritic cells. European journal of immunology 1995, 25(9):2668-2672

20. Locksley RM, Reiner SL, Hatam F, Littman DR, Killeen N: Helper T cells without CD4: control of leishmaniasis in CD4-deficient mice. Science 1993, 26I(5 I 27): | 1448-I45I.

21. Elloso MM, Scott P: Expression and contribution of B7-I (CD80) and B7-2 (CD86) in the early immune response to Leishmania major infection. I Immunol 1999, I 62(II):6708-67|5

22. Favali C, Costa D, Afonso L, Conceicao V, Rosato A, Oliveira F, Costa J, Barral A, Barral-Netto M, Brodskyn Cl: Role of costimulatory molecules in immune response of patients with cutaneous leishmaniasis. Microbes Infect 2005, 7(1):86-92.
23. Machado P, Kanitakis J, Almeida R, Chalon A, Araujo C, Carvalho EM: Evidence of in situ cytotoxicity in American cutaneous leishmaniasis. Eur J Dermatol 2002, I 2(5):449-45I.

24. Antonelli LR, Dutra WO, Almeida RP, Bacellar O, Carvalho EM, Gollob KJ: Activated inflammatory $\mathbf{T}$ cells correlate with lesion size in human cutaneous leishmaniasis. Immunology letters 2005, I 0 I(2):226-230.

25. Brodie TM, Smith MC, Morris RV, Titus RG: Immunomodulatory Effects of the Lutzomyia longipalpis Salivary Gland Protein Maxadilan on Mouse Macrophages. Infect Immun 2007, 75(5):2359-2365

26. Scott P: The role of THI and TH2 cells in experimental cutaneous leishmaniasis. Exp Parasitol 1989, 68(3):369-372.

27. Diehl S, Rincon M: The two faces of IL-6 on Th I/Th2 differentiation. Molecular immunology 2002, 39(9):53I-536.

28. Laskay T, van Zandbergen G, Solbach W: Neutrophil granulocytes--Trojan horses for Leishmania major and other intracellular microbes? Trends Microbiol 2003, I I (5):2 I 0-2 I 4 .

29. Belkaid Y, Mendez S, Lira R, Kadambi N, Milon G, Sacks D: A natural model of Leishmania major infection reveals a prolonged "silent" phase of parasite amplification in the skin before the onset of lesion formation and immunity. J Immunol 2000, 165(2):969-977.

30. Pompeu MM, Brodskyn C, Teixeira MJ, Clarencio J, Van Weyenberg J, Coelho IC, Cardoso SA, Barral A, Barral-Netto M: Differences in gamma interferon production in vitro predict the pace of the in vivo response to Leishmania amazonensis in healthy volunteers. Infect Immun 200I, 69( I 2):7453-7460.

31. Soares MB, Titus RG, Shoemaker CB, David JR, Bozza M: The vasoactive peptide maxadilan from sand fly saliva inhibits TNF-alpha and induces IL-6 by mouse macrophages through interaction with the pituitary adenylate cyclase-activating polypeptide (PACAP) receptor. I Immunol 1998, 160(4): $181 \mid-1816$.

32. Titus RG, Bishop JV, Mejia JS: The immunomodulatory factors of arthropod saliva and the potential for these factors to serve as vaccine targets to prevent pathogen transmission. Parasite Immunol 2006, 28(4): |3|-|4|.

33. Modi GB, Tesh RB: A simple technique for mass rearing Lutzomyia longipalpis and Phlebotomus papatasi (Diptera: Psychodidae) in the laboratory. J Med Entomol 1983, 20(5):568-569.

34. Castilho TM, Shaw J], Floeter-Winter LM: New PCR assay using glucose-6-phosphate dehydrogenase for identification of Leishmania species. J Clin Microbiol 2003, 4 I (2):540-546.

35. McMahon-Pratt D, Bennett E, David JR: Monoclonal antibodies that distinguish subspecies of Leishmania braziliensis. J Immunol I 982, I 29(3):926-927.

Publish with Biomed Central and every scientist can read your work free of charge

"BioMed Central will be the most significant development for disseminating the results of biomedical research in our lifetime. "

Sir Paul Nurse, Cancer Research UK

Your research papers will be:

- available free of charge to the entire biomedical community

- peer reviewed and published immediately upon acceptance

- cited in PubMed and archived on PubMed Central

- yours - you keep the copyright 